\title{
In Vitro Evaluation of Aerosol Delivery by Different Nebulization Modes in Pediatric and Adult Mechanical Ventilators
}

\author{
Gwo-Hwa Wan PhD, Hui-Ling Lin MSc RRT RN FAARC, James B Fink PhD RRT FAARC, \\ Yen-Hey Chen MSc, Wei-Jhen Wang RRT, Yu-Chun Chiu RRT, \\ Yu-Yao Kao RRT, and Chia-Jung Liu RRT
}

\begin{abstract}
BACKGROUND: Aerosol delivery through mechanical ventilation is influenced by the type of aerosol generator, pattern of nebulization, and a patient's breathing pattern. This study compares the efficiency of pneumatic nebulization modes provided by a ventilator with adult and pediatric in vitro lung models. METHODS: Three pneumatic nebulization modes (inspiratory intermittent [IIM], continuous [CM], and expiratory intermittent [EIM]) provided by the Galileo Gold ventilator delivered medical aerosol to collection filters distal to an endotracheal tube with adult and pediatric test lungs. A unit dose of $5 \mathrm{mg} / 2.5 \mathrm{~mL}$ albuterol was diluted into $4 \mathrm{~mL}$ with distilled water and added to a jet nebulizer. The nebulizer was placed proximal to the ventilator, $15 \mathrm{~cm}$ from the inlet of the heated humidifier chamber with a T-piece and corrugated aerosol tubing and powered by gas from the ventilator in each of the 3 modes. Time for nebulization was recorded in minutes. Albuterol samples collected in the inhalation filter, nebulizer, T-piece, and corrugated tubing were eluted with distilled water and analyzed with a spectrophotometer. RESULTS: The inhaled drug, as a percentage of total dose in both lung models, was 5.1-7.5\%, without statistical significance among the 3 modes. Median nebulization times for IIM, CM, and EIM were 38.9, 14.3, and $17.7 \mathrm{~min}$, respectively, and nebulization time for the 3 modes significantly differed $(P<.001)$. The inhaled drug mass for the 3 modes with the adult lung model was similar to that with the pediatric lung model $(7.39 \pm 0.76$ vs $6.27 \pm 0.69 \%, P=.77)$. CONCLUSIONS: Aerosol drug delivery with a jet nebulizer placed proximal to the ventilator was not dependent on nebulization mode during simulated pediatric and adult conventional mechanical ventilation. Use of expiratory intermittent mode and continuous nebulization should be considered to reduce treatment time. Key words: aerosol drug delivery; bronchodilator; small-volume nebulizer; endotracheal tube; mechanical ventilation; nebulization mode. [Respir Care 2014;59(10):1494-1500. (C) 2014 Daedalus Enterprises]
\end{abstract}

\section{Introduction}

Aerosolized bronchodilators are commonly used to treat mechanically ventilated patients with airway disease and

\footnotetext{
Dr Wan, Ms Lin, and Ms Chen are affiliated with the Department of Respiratory Therapy, College of Medicine, Chang Gung University, Taoyuan, Taiwan. Dr Fink is affiliated with the Division of Respiratory Therapy, Georgia State University, Atlanta, Georgia. Ms Wang is affiliated with the Department of Respiratory Therapy, National Taiwan University Hospital, Taipei, Taiwan. Ms Chiu is affiliated with the Graduate Institute of Pharmacology, College of Medicine, National Taiwan University, Taipei, Taiwan. Mr Kao and Ms Liu are affiliated with the
}

conditions. The jet nebulizer is frequently used to administer bronchodilators and other agents during mechanical ventilation.

\footnotetext{
Department of Respiratory Therapy, Chang Gung Memorial HospitalLinko Branch, Taoyuan, Taiwan.

Ms Lin presented a version of this paper at the Open Forum of the AARC Congress 2012, held November 10-13, 2012, in New Orleans, Louisiana.

Dr Fink is a consultant to Aerogen, Bayer, Boehringer Ingelheim, Cubist Pharmaceuticals, Dance Biopharm, Novartis, ONY, Parion, Aridis Pharmaceuticals, and the World Health Organization. The other authors have disclosed no conflicts of interest.
} 
The efficiency of drug delivery through mechanical ventilation in adults is enhanced by many factors, including CPAP, mode (spontaneous breathing), ${ }^{1}$ longer duty cycle and inspiratory time, ${ }^{2}$ lower inspiratory flow, ${ }^{3}$ and lower bias flow. ${ }^{1,2,4-6}$ Aerosol delivery varies with different types of aerosol generators, brands of nebulizer used, and their position in the ventilator circuit. ${ }^{6-8}$ Other authors reported that the higher the peak inspiratory flow, the greater the impact of inspiratory waveform on aerosol delivery. ${ }^{5}$

Furthermore, factors improving aerosol delivery through mechanical ventilation in pediatric patients include larger endotracheal tubes (ETTs), use of metered-dose inhalers with spacers, synchronization with inspiration, use of heliox (80:20 helium-oxygen mixture) as a gas power source, and positioning a jet nebulizer distal to the ventilator circuit. ${ }^{8-12}$

More recently, researchers have reported greater aerosol delivery with a continuous jet nebulizer placed proximal to the ventilator and before the humidifier in both adult and pediatric lung models. ${ }^{11,13} \mathrm{~A}$ number of benefits have been proposed to placing jet nebulizers proximal to the ventilator rather than in the more conventional placement in the inspiratory limb or at the Y-piece near the patient. Placement of the nebulizer in the inspiratory limb of the ventilator circuit proximal to the ventilator allows the nebulizer to be oriented in an optimal position that does not change with turning the patient and eliminates the weight of the nebulizer near the patient airway. Placement at the inlet of the humidifier reduces access of patient secretions and condensate in the inspiratory limb of the ventilator circuit to contaminate the nebulizer. In addition, passing aerosol through the humidifier allows larger particles to rain out, reducing the mass of aerosol that might deposit on a sensor placed between the ventilator circuit and the patient airway as well as condensate that might form in the inspiratory limb.

With those potential benefits in mind, it is important to quantify aerosol drug delivery distal to the ETT with all modes. Two basic aerosol generation scenarios have been described with adult mechanical ventilation: generating aerosol only during inspiration and generating aerosol continuously throughout the respiratory cycle. Some ventilators provide a nebulization function that synchronizes aerosol generation during mechanical ventilation. Over the last $20 \mathrm{y}$, reports of benefits with synchronized over continuous nebulization have been mixed, depending on the placement of the nebulizer. A study comparing aerosol delivery

Correspondence: Hui-Ling Lin MSc RRT RN FAARC. E-mail: huilingrrt@gmail.com.

DOI: $10.4187 /$ respcare.02999

\section{QUICK LOOK}

\section{Current knowledge}

Aerosol delivery during mechanical ventilation can be influenced by the aerosol generator, pattern of nebulization, presence of humidification, ventilator settings, and patient breathing pattern. The pattern of nebulization includes continuous, synchronized with inspiration, and during expiration.

\section{What this paper contributes to our knowledge}

In a lung model study, aerosolized drug delivery with a jet nebulizer placed proximal to the ventilator was not affected by the pattern of nebulization. However, synchronized inspiratory nebulization required a 3 -fold longer nebulization time.

from a jet nebulizer synchronized with inspiration compared with continuous nebulization reported a nearly 2-fold increase in delivery with jet nebulizer placement at the Y-piece, but only $15 \%$ greater compared with placement in the middle of the inspiratory limb (or manifold position). ${ }^{14}$ In contrast, a reduction with synchronized nebulization at the manifold position has been reported. ${ }^{15}$ Early in vivo deposition studies reported $<3 \%$ deposition utilizing jet nebulization synchronized with inspiration. ${ }^{16} \mathrm{~A}$ study by Miller et $\mathrm{al}^{6}$ compared breath-actuated nebulization by 2 different ventilators (PB7200, Puritan Bennett, Pleasanton, California; and Dräger, Lübeck, Germany). They reported substantial improvements with synchronized versus continuous nebulization with the nebulizer placed in the inspiratory limb at the Y-piece.

Di Paolo et $\mathrm{al}^{17}$ and Sidler-Moix et al ${ }^{18}$ compared different nebulization modes in an infant bench model and reported better aerosol delivery with nebulization synchronized during expiration compared with inspiration or continuously throughout the cycle. They reported that a jet nebulizer delivered more efficiently with intermittent expiratory mode on a ventilator (Galileo, Hamilton Medical, Bonaduz, Switzerland).

This in vitro study compares the influence of 3 pneumatic nebulization modes of a ventilator using adult and pediatric ventilator parameters and lung models. We hypothesized that aerosol generated with the 3 modes during mechanical ventilation would deliver a similar drug dose to the distal end of an ETT. We also hypothesized that aerosol delivery would be greater with adult than pediatric settings. The study was performed in the Respiratory Therapy Research Laboratory at Chang Gung University. 


\section{Methods}

\section{Lung Model}

Figure 1 shows the experimental apparatus of the lung model. A ventilator (Galileo Gold, Hamilton Medical) was set to deliver adult parameters (tidal volume $\left[\mathrm{V}_{\mathrm{T}}\right]$ of $600 \mathrm{~mL}$, breathing frequency of 16 breaths/min, PEEP $5 \mathrm{~cm} \mathrm{H}_{2} \mathrm{O}$, and inspiration-expiration ratio [I:E] 1:2.5) and pediatric parameters $\left(\mathrm{V}_{\mathrm{T}} 160 \mathrm{~mL}\right.$, breathing frequency of 25 breaths/ min, PEEP $5 \mathrm{~cm} \mathrm{H}_{2} \mathrm{O}$, and $\left.\mathrm{I}: \mathrm{E} 1: 2\right)$. A 120 -cm ventilator circuit connected the ventilator to a test lung (TTL, Michigan Instruments, Grand Rapids, Michigan) via an ETT (7.5-mm [adult] and 5.0-mm [pediatric] inner diameter) positioned distal to a Y-piece and a flow sensor. Adult (22- $\mathrm{mm}$ inner diameter) and pediatric (15-mm inner diameter) ventilator circuits were used during the experiments. A jet nebulizer (GaleMed Corp, Taipei, Taiwan) was placed proximal to the ventilator $15 \mathrm{~cm}$ from the inlet of a heated pass-over humidifier (MR850, Fisher \& Paykel Healthcare, Auckland, New Zealand) operated at $37^{\circ} \mathrm{C}$. Aerosols were collected distal to the ETT by a hydrophobic bacterial filter (GaleMed Corp).

\section{Formulation and Aerosol Delivery}

A unit dose of salbutamol (known as albuterol and Ventolin Nebules [5 mg/2.5 mL], GlaxoSmithKline, Philadelphia, Pennsylvania) was diluted with distilled water to a total fill volume of $4 \mathrm{~mL}$. The nebulizer was powered by the ventilator in 3 nebulization modes: (1) inspiratory intermittent nebulization mode (IIM) synchronized with inspiration phase, (2) continuous nebulization mode (CM), and (3) expiratory intermittent nebulization mode (EIM) during the expiration phase. Albuterol delivery from the nebulizer was stopped 5 breathing cycles after the onset of sputter. Six new nebulizers were used for testing with each scenario.

\section{Sample Measurement}

Albuterol deposited on the filters, T-pieces, and corrugated tubing between the nebulizer and heater, as well as the residual dose in nebulizers at the end of dosing, was eluted with distilled water and analyzed by a spectrophotometer (BioMate 3S UV-visible, Thermo Fisher Scientific, Waltham, Massachusetts) at $276 \mathrm{~nm}$ to quantify drug mass, which was reported as a percentage of total initial dose placed in the nebulizer. Nebulization time from initiation of aerosol generation to 5 breaths after onset of sputter was recorded in minutes.

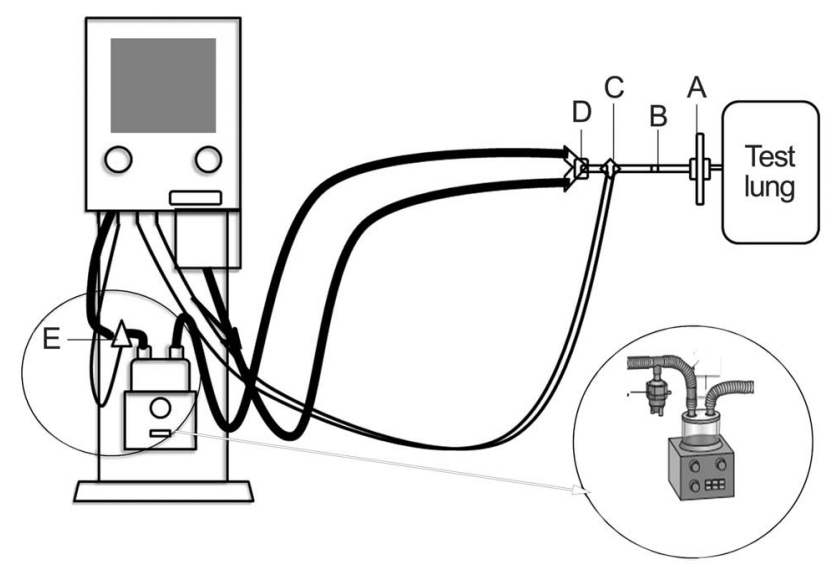

Fig. 1. Diagram of experimental apparatus. A jet nebulizer (E) powered by the ventilator nebulization function was placed in the ventilator outlet $15 \mathrm{~cm}$ from the heater, and a filter for aerosol collection (A) was placed distal to the endotracheal tube (B). Also shown are the flow sensor (C) and the Y-piece (D).

\section{Statistical Analysis}

Statistical analyses were performed using SPSS 18.0 (SPSS, Chicago, Illinois). Figures were constructed with Prism 5.0 (GraphPad Software, San Diego, California). Significance was set at $P=.05$. Descriptive statistics were analyzed, and eluted drug amounts were converted to percentage of total dose. One-way analysis of variance with Bonferroni post hoc comparisons for normally distributed data was used to assess the magnitude of group differences for continuous variables. The Kruskal-Wallis test and Mann-Whitney U test for non-normally distributed data were used to identify group differences in nebulization time.

\section{Results}

\section{Drug Delivery}

With the adult model, drug delivery on the inhalation filter distal to the ETT expressed as a percentage of total dose was between 7.7 and $7.0 \%$ of the charged dose in 3 nebulization pattern modes $(P=.99)$ (Fig. 2A). In contrast, with the pediatric model, drug delivered by IIM trended higher than $\mathrm{CM}$ and EIM, without achieving statistical significance $(P=.40)$ (Fig. 2B). The average percentage of inhaled dose was similar with all 3 nebulization modes with both adult and pediatric lung models (7.39 \pm 0.76 and $6.27 \pm 0.69 \%$, respectively, $P=.77$ ). Comparing the drug deposited on the inhaled filter among the 3 modes in the pediatric and adult lung models, there were no significant differences $(P=.2$ for IIM, $P=.67$ for EIM, and $P=.09$ for $\mathrm{CM}$ ). 


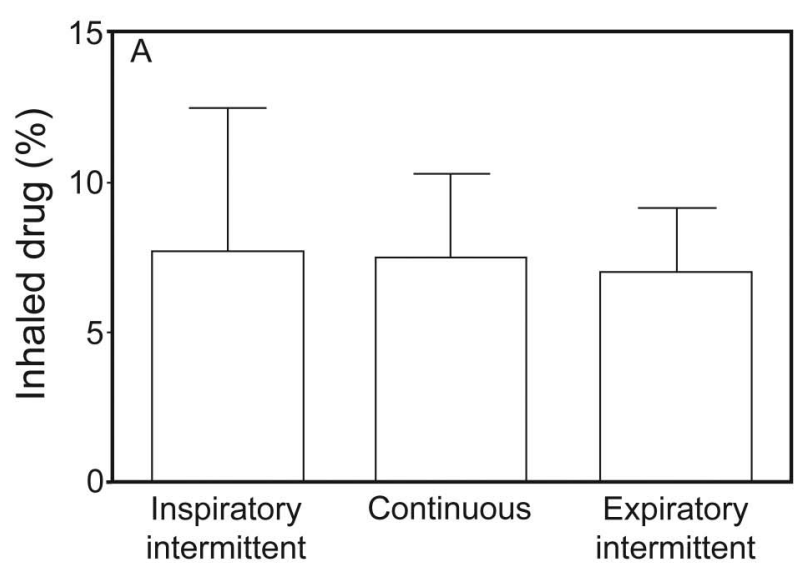

Mode of nebulization

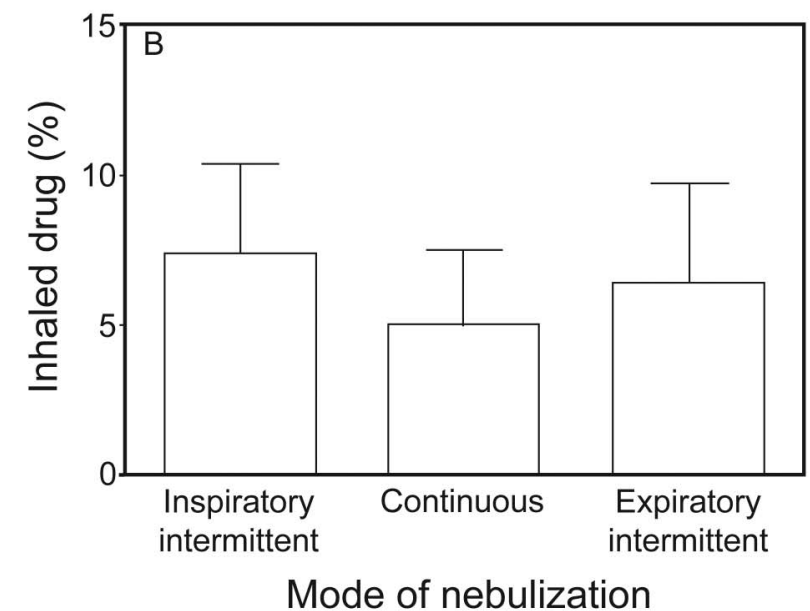

Fig. 2. Comparison of inhaled drug mass among 3 modes of nebulization. A: In an adult lung model. B: In a pediatric lung model.

Table 1 lists the residual drug in nebulizers and on the accessory tubes (T-pieces and corrugated tubing) expressed as a percentage of total drug dose (mean \pm SD) for the adult and pediatric lung models. The drug deposited on accessory tubes was greater in IIM than in CM or EIM $(P<.001$ and $P=.04$, respectively). In contrast, in the pediatric lung model, no statistical difference existed in the amount of albuterol deposited in the nebulizer and on the accessory tubes in any mode.

\section{Nebulization Time}

The median nebulization times were 37.0 (interquartile range [IQR], 33.3-42.7) $\mathrm{min}$ for IIM, 14.0 (IQR 11.714.6) $\mathrm{min}$ for CM, and 17.7 (IQR 16.1-22.4) min for EIM with the adult lung model. A clear difference existed in nebulization time among the 3 modes in the adult lung model $(P<.001)$ (Fig. 3A). Additionally, in the pediatric lung model, median nebulization times were 34.8 (IQR 33.0-44.8) min for IIM, 13.84 (IQR 11.8-15.2) min for
Table 1. Drug as Percentage of Total Dose Deposited in Nebulizer and Ventilator Accessory Tubing at End of Aerosol Administration With 3 Nebulization Modes During Mechanical Ventilation With Adult and Pediatric Lung Models

\begin{tabular}{|c|c|c|c|c|}
\hline \multirow[b]{2}{*}{ Deposition } & \multicolumn{3}{|c|}{ Mode } & \multirow[b]{2}{*}{$P$} \\
\hline & $\begin{array}{c}\text { IIM } \\
(n=6)\end{array}$ & $\begin{array}{c}\text { CM } \\
(n=6)\end{array}$ & $\begin{array}{c}\text { EIM } \\
(n=6)\end{array}$ & \\
\hline \multicolumn{5}{|l|}{ Adult lung model } \\
\hline Nebulizer, \% & $40.7 \pm 1.8$ & $40.3 \pm 1.6$ & $41.6 \pm 1.9$ & .89 \\
\hline $\begin{array}{l}\text { Accessory tubes, } \\
\%\end{array}$ & $9.6 \pm 0.1$ & $6.3 \pm 0.4^{*}$ & $5.6 \pm 0.7 \dagger$ & $<.001$ \\
\hline \multicolumn{5}{|l|}{ Pediatric lung model } \\
\hline Nebulizer, \% & $36.3 \pm 6.2$ & $41.9 \pm 2.5$ & $40.5 \pm 2.3$ & .66 \\
\hline $\begin{array}{l}\text { Accessory tubes, } \\
\%\end{array}$ & $6.1 \pm 0.7$ & $4.7 \pm 0.6$ & $4.4 \pm 0.4$ & .11 \\
\hline \multicolumn{5}{|c|}{$\begin{array}{l}\text { Values are mean } \pm \mathrm{SD} \text {. } \\
* P<.05 \text {, comparison between IIM and CM } \\
\dagger P<.05 \text {, comparison between IIM and EIM } \\
\text { IIM }=\text { inspiratory intermittent mode } \\
\mathrm{CM}=\text { continuous mode } \\
\text { EIM }=\text { expiratory intermittent mode }\end{array}$} \\
\hline
\end{tabular}

CM, and 18.8 (IQR 17.5-22.0) min for EIM $(P<.001)$ (Fig. 3B). Additionally, nebulization times with the adult lung model $(23.2 \pm 2.7 \mathrm{~min})$ and pediatric lung model (23.4 $\pm 2.6 \mathrm{~min}, P=.79)$ were similar.

\section{Discussion}

Our results demonstrated similar aerosol delivery efficiency for all 3 nebulization modes from a jet nebulizer placed proximal to the ventilator during mechanical ventilation with both adult and pediatric models. With similar drug delivery in all 3 modes with the adult model and only marginally greater delivery with IIM in the pediatric model, it appears that all modes should be considered viable for aerosol administration. However, the much longer nebulization time with IIM for both adult and pediatric models is of interest and may be of clinical importance.

Our findings are consistent with those of Berlinski and Willis, ${ }^{11}$ who evaluated delivery of albuterol from jet nebulizers operated continuously at the ventilator (prehumidifier) ranging from 3.6 to $7 \%$ compared with $<2 \%$ with placement near the patient airway. Using a pediatric model of mechanical ventilation, Ari et $\mathrm{al}^{13}$ reported deposition at the ventilator of $4.1-5.2$ versus $3.8-4.2 \%$ at the Y-piece, with a range of $4.7-5.2$ versus $4.0-4.7 \%$ in the adult model.

Sidler-Moix et al ${ }^{18}$ evaluated the influences of jet nebulizer brands and nebulization modes and positions provided by the Galileo ventilator with the humidification system off during nebulization in pediatric lung models. They reported a 3-fold greater drug delivery in EIM $(\sim 6 \%)$ with the aerosol generator placed proximal to the ventila- 


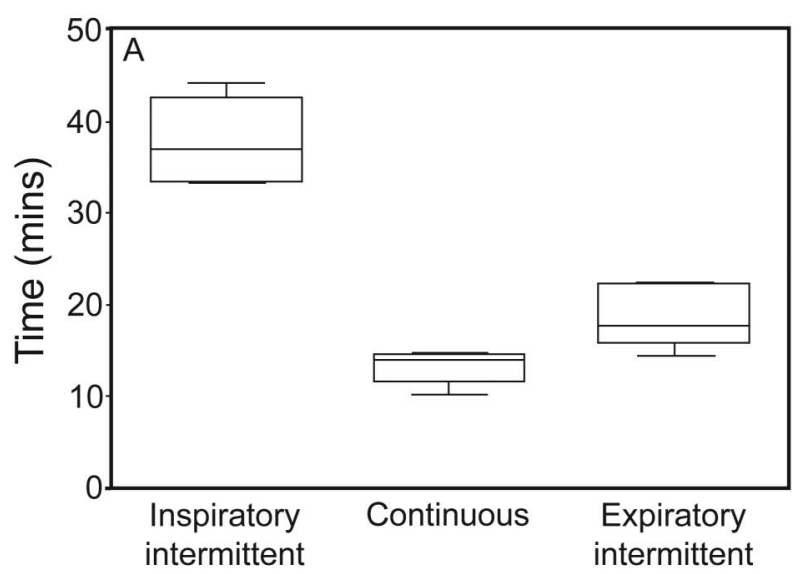

Mode of nebulization

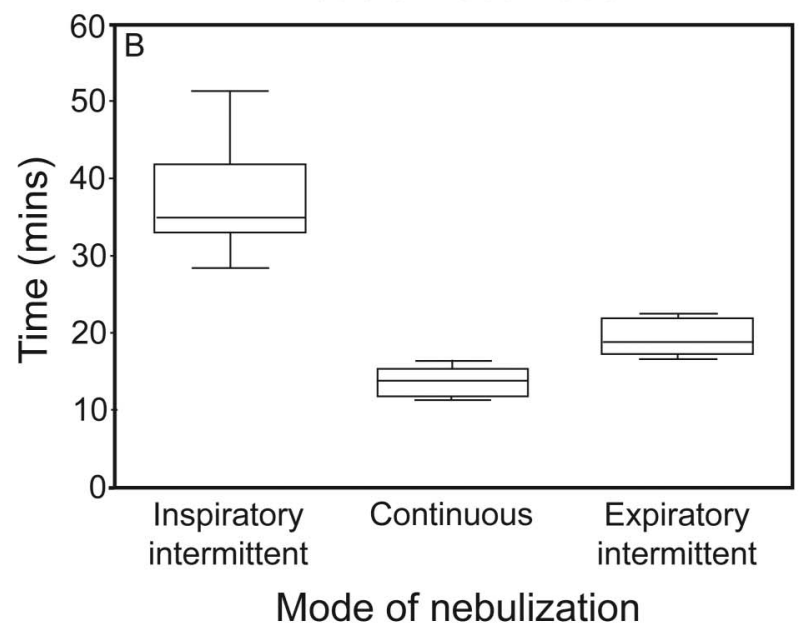

Fig. 3. Comparison of nebulization time among 3 modes of nebulization. A: In an adult lung model. B: In a pediatric lung model. The inspiratory intermittent modes had $P<.001$ among the 3 nebulization modes. Whiskers show maximums and minimums, the center box represents the interquartile range, and the center line denotes the 50th percentile.

tor compared with IIM (2\%) and CM (4\%) with the nebulizer placed near the patient. Their similar inspiratory time of $0.8 \mathrm{~s}$ and expiratory time of $1.6 \mathrm{~s}$ (I:E 1:2) provided 2 -fold more time available to generate aerosol during expiration, which served to charge the ventilator circuit, resulting in more aerosol inhaled. They compared different types of nebulizers as well as different positions with those 3 nebulization modes and reported that drug delivery was negatively correlated to the time required to reach the recommended gas flow for a nebulizer. ${ }^{18} \mathrm{Al}-$ though we found similar levels of albuterol deposited in the inspiratory filter during EIM $(\sim 6 \%)$ with the aerosol generator proximal to the ventilator, IIM and CM deposition was greater with the proximal position than with placement near the airway.

The pediatric ventilator circuit (15-mm inner diameter) has a smaller internal volume than the adult circuit (22-mm inner diameter), possibly resulting in the trend for higher delivery with IIM in the pediatric model compared with the adult model. The actual aerosol generation time is similar in each mode between the adult and pediatric models. The lower circuit volume with the pediatric model likely acts to reduce the reservoir effect with the continuous gas flow through the nebulizer. The dynamics of the fate of aerosol are different between modes but appear consistent in each mode for the same time frames of aerosol generation.

The compressor of the Galileo ventilator generates a pressure of 900 mbar during each nebulization. Di Paolo et al, ${ }^{17}$ who assessed the pressure-flow relationship, found that IIM was the least efficient, with lower maximum pressure and lower flow than CM or EIM. They tested flows provided to a jet nebulizer, which varied between 5.5 and 8.7 L/min. Additionally, they confirmed that the high internal resistance of the nebulizer used resulted in prolonged time to achieve the recommended flow of $5 \mathrm{~L} / \mathrm{min}$. One nebulizer took $0.55 \mathrm{~s}$ to reach $5 \mathrm{~L} / \mathrm{min}$ when the inspiratory time was set at $0.8 \mathrm{~s}$; thus, the nebulization time of each breath was as short as $0.25 \mathrm{~s}$. The pediatric lung model in this study was set to 25 breaths/min with an inspiratory time of $0.8 \mathrm{~s}$. These settings resulted a median nebulization time of $34.8 \mathrm{~min}$. Because of the differences in the breathing frequency between pediatric and adult models, the calculated inspiratory times were 20 and $17.6 \mathrm{~s}$, respectively, with expiratory times per min of 40 and $43 \mathrm{~s}$, respectively. This may explain the similar inhaled dose between models within each mode of nebulization in both breathing patterns.

Goode et al ${ }^{12}$ placed a jet nebulizer (AeroTech II at $6 \mathrm{~L} / \mathrm{min}$ ) in the inspiratory limb of an adult ventilator circuit, with only one set of ventilator parameters used. Aerosol was administered with continuous jet nebulization, resulting in delivery efficiencies of 1.2-2.4\% with $\mathrm{O}_{2}$ and $\mathrm{HeO}_{2}$, respectively. Ari et $\mathrm{al}^{13}$ reported deposition of $3.6 \%$ from the jet nebulizer placed in the inspiratory limb of a humidified circuit using a nebulizer (Airlife MistyNeb, Allegiance Healthcare Corp, McGaw Park, Illinois) operated continuously at $8 \mathrm{~L} / \mathrm{min}$ gas, $\mathrm{V}_{\mathrm{T}} 500 \mathrm{~mL}$, breathing frequency of 15 breaths/min, and peak inspiratory flow of $60 \mathrm{~L} / \mathrm{min}$, with deposition increasing to $\sim 6 \%$ when the jet nebulizer was moved proximal to the ventilator. Our study, with a $\mathrm{V}_{\mathrm{T}} 600 \mathrm{~mL}$, breathing frequency of 16 breaths/ min, and I:E 1:2.5, using an adult circuit and a different nebulizer produced results similar to those obtained by Ari et $\mathrm{al}^{13}$ in the proximal position. None of the 3 studies isolated I:E as a variable. Different nebulizers, ventilator parameters, and measurements in the past $30 \mathrm{y}$ can impact quantification of delivery efficiency.

Clinically, as nebulizer treatment time increases, the likelihood that aerosolized drug therapy will be interrupted by other clinical practices increases. IIM delivered a sim- 
ilar inhaled drug mass compared with the other 2 nebulization modes, yet drug delivery by IIM required a much longer nebulization time. The use of CM and EIM can be used for routine nebulization treatment for mechanically ventilated patients in hospitals. If time of administration is considered a valued resource, CM and EIM nebulization would be preferable to IIM.

The nebulizer in this study was placed $15 \mathrm{~cm}$ proximal to the inlet of the heated humidifier chamber, similar to that in previous studies. ${ }^{8,11,13,19}$ Recently, Berlinski and Willis ${ }^{11}$ demonstrated that placing a jet nebulizer proximal to the ventilator circuit, both before and after the humidifier, increases drug delivery in a pediatric circuit.

We were surprised to find such similar deposition in all 3 modes in both the adult and pediatric models. Our study indicates that the inhaled mass was similar for the adult and pediatric lung models in all modes studied, likely because both models had a large internal circuit volume acting as a reservoir, including the ventilator circuit and heated humidification chamber. Thus, we suggest placing a jet nebulizer far from the Y-piece of a ventilator circuit to decrease its influence on aerosol delivery.

The total inspiratory and expiratory times with both lung models were similar, resulting in similar inhaled doses. A considerable amount of aerosol generated in all 3 modes resides in the inspiratory limb and heater reservoir between breaths. The smaller volume of the pediatric circuit would allow the same nebulizer gas flow to wash a greater proportion of the generated aerosol through the circuit, resulting in similar delivery between the 2 models.

Analytical results show that drug deposition on the Tpiece and corrugated tubing by IIM was clearly higher than that by the other 2 modes. The T-piece is the first impaction site when aerosols are generated by a jet nebulizer. During the inspiratory phase, the aerosol clouds meet the gas flow at the T-piece and corrugated tubing. The gas flow enhances first impaction; consequently, drug delivery was highest with IIM. During the experiment, the ventilator waveform showed that the inspiratory flows were approximately $34 \mathrm{~L} / \mathrm{min}$ for the adult lung model and $12 \mathrm{~L} / \mathrm{min}$ for the pediatric lung model. Higher amounts of albuterol were deposited in the T-piece and corrugated tubing in the adult lung model than in the pediatric lung model.

A limitation of this study is that we compared only one set of ventilator parameters for both adult and pediatric lung models (based on physiologic parameters). Further evaluation with a broader range of ventilator modes and parameters and types of nebulizers was beyond the scope of this study but would add greater insight on the impact of nebulization modes. Further investigations with various breathing patterns are warranted. Because this was an in vitro study, clinical studies of mechanically ventilated patients would help establish whether these in vitro results are clinically relevant.

This study was limited to one brand and model of ventilator. Variations between ventilators may yield different results. However, based on our finding, unless proven otherwise, paying extra to have a ventilator that allows synchronized nebulization might not be worth the expense. Clinically, nebulization is terminated either at onset of sputtering or when no aerosols are generated. ${ }^{20}$ Sputter is usually detected via audible and/or visual perceptions, which may differ among health professionals. We terminated nebulization at 5 breaths post-onset of sputter. Although reports suggest that minimum additional aerosol is delivered post-onset of sputter, this method may result in underestimation of absolute dose if nebulization was run to dryness; however, because the determination of sputter was applied across all nebulization modes, the relative differences in inhaled dose should remain relevant.

\section{Conclusions}

Aerosol drug delivery with a jet nebulizer placed proximal to the ventilator was not dependent on nebulization mode provided by the Galileo ventilator during simulated conventional pediatric and adult subject ventilation. The synchronized inspiratory nebulization mode provided similar drug delivered distal to the ETT compared with the other 2 modes but required a 3 -fold longer nebulization time. Use of EIM and CM nebulization should be considered to reduce treatment time.

\section{REFERENCES}

1. Coleman DM, Kelly HW, McWilliams BC. Determinants of aerosolized albuterol delivery to mechanically ventilated infants. Chest 1996;109(6):1607-1613.

2. Dhand R, Tobin MJ. Bronchodilator delivery with metered-dose inhalers in mechanically-ventilated patients. Eur Respir J 1996;9(3): 585-595.

3. Fink JB, Dhand R, Grychowski J, Fahey PJ, Tobin MJ. Reconciling in vitro and in vivo measurements of aerosol delivery from a metered-dose inhaler during mechanical ventilation and defining efficiency-enhancing factors. Am J Respir Crit Care Med 1999;159(1): 63-68.

4. Fink JB, Dhand R, Duarte AG, Jenne JW, Tobin MJ. Aerosol delivery from a metered-dose inhaler during mechanical ventilation. An in vitro model. Am J Respir Crit Care Med 1996;154(2):382-387.

5. Hess DR, Dillman C, Kacmarek RM. In vitro evaluation of aerosol bronchodilator delivery during mechanical ventilation: pressure-control vs. volume control ventilation. Intensive Care Med 2003;29(7): 1145-1150.

6. Miller DD, Amin MM, Palmer LB, Shah AR, Smaldone GC. Aerosol delivery and modern mechanical ventilation: in vitro/in vivo evaluation. Am J Respir Crit Care Med 2003;168(10):1205-1209.

7. O'Riordan TG, Weinstein MD, Mao Y. Bench testing of nebulizers: a comparison of three methods. J Aerosol Med 1999;12(2):59-66.

8. Ari A, Atalay OT, Harwood R, Sheard MM, Aljamhan EA, Fink JB. Influence of nebulizer type, position, and bias flow on aerosol drug 


\section{Aerosol Delivery by Different Ventilator Nebulization Modes}

delivery in simulated pediatric and adult lung models during mechanical ventilation. Respir Care 2010;55(7):845-851.

9. Garner SS, Wiest DB, Bradley JW. Albuterol delivery by metereddose inhaler with a pediatric mechanical ventilatory circuit model. Pharmacotherapy 1994;14(2):210-214.

10. Pelkonen AS, Nikander K, Turpeinen M. Jet nebulization of budesonide suspension into a neonatal ventilator circuit: synchronized versus continuous nebulizer flow. Pediatr Pulmonol 1997;24(4):282-286.

11. Berlinski A, Willis JR. Albuterol delivery by 4 different nebulizers placed in 4 different positions in a pediatric ventilator in vitro model. Respir Care 2013;58(7):1124-1133.

12. Goode ML, Fink JB, Dhand R, Tobin MJ. Improvement in aerosol delivery with helium-oxygen mixtures during mechanical ventilation. Am J Respir Crit Care Med 2001;163(1):109-114.

13. Ari A, Areabi H, Fink JB. Evaluation of aerosol generator devices at 3 locations in humidified and non-humidified circuits during adult mechanical ventilation. Respir Care 2010;55(7):837-844.

14. Hughes JM, Saez J. Effects of nebulizer mode and position in a mechanical ventilator circuit on dose efficiency. Respir Care 1987; 32(12):1131-1135.
15. Quinn WW. Effect of a new nebulizer position on aerosol delivery during mechanical ventilation: a bench study. Respir Care 1992; 37(5):423-431

16. MacIntyre NR, Silver RM, Miller CW, Schuler F, Coleman RE. Aerosol delivery in intubated, mechanically ventilated patients. Crit Care Med 1985;13(2):81-84

17. Di Paolo ER, Pannatier A, Cotting J. In vitro evaluation of bronchodilator drug delivery by jet nebulization during pediatric mechanical ventilation. Pediatr Crit Care Med 2005;6(4):462-469.

18. Sidler-Moix AL, Dolci U, Berger-Gryllaki M, Pannatier A, Cotting J, Di Paolo ER. Albuterol delivery in an in vitro pediatric ventilator lung model: comparison of jet, ultrasonic, and mesh nebulizers. Pediatr Crit Care Med 2013;14(2):e98-e102.

19. Moraine JJ, Truflandier K, Vandenbergen N, Berré J, Mélot C, Vincent JL. Placement of the nebulizer before the humidifier during mechanical ventilation: effect on aerosol delivery. Heart Lung 2009;38(5):435-439.

20. Dolovich MB, Ahrens RC, Hess DR, Anderson P, Dhand R, Rau JL, et al. Device selection and outcomes of aerosol therapy: evidence-based guidelines: American College of Chest Physicians/American College of Asthma, Allergy, and Immunology. Chest 2005;127(1):335-371.

This article is approved for Continuing Respiratory Care Education credit. For information and to obtain your CRCE

(free to AARC members) visit

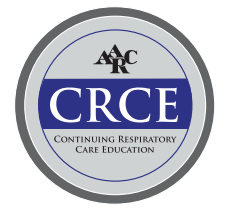

